\title{
Effects of voluntary exercise on tumorigenesis in the C3(1)/SV40Tag transgenic mouse model of breast cancer
}

\author{
J.L. STEINER ${ }^{1,2}$, J.M. DAVIS ${ }^{2}$, J.L. McCLELLAN ${ }^{1,2}$, R.T. ENOS ${ }^{1,2}$ and E.A. MURPHY ${ }^{1}$ \\ ${ }^{1}$ Department of Pathology, Microbiology and Immunology, School of Medicine at the University of South Carolina, \\ Columbia, SC 29209; ${ }^{2}$ Department of Exercise Science, University of South Carolina, Columbia, SC 29208, USA
}

Received December 3, 2012; Accepted January 16, 2013

DOI: 10.3892/ijo.2013.1827

\begin{abstract}
Epidemiologic studies suggest an association between physical activity (PA) and breast cancer risk. We examined the relationship between voluntary wheel running and breast cancer in C3(1)/SV40Tag mice. Female FVB/N and C3(1)/SV40Tag mice were assigned to either PA [C3(1)-PA] $(n=12)$ or sedentary (Sed) [C3(1)-Sed] $(n=15)$ treatment and were placed in a cage with access to a running wheel (PA) or without (Sed) from 4 to 24 weeks of age (sacrifice). Physical activity data were analyzed for running distance, time and speed. Body composition was examined at 12 weeks of age. Tumors were counted twice weekly and at sacrifice to assess multiplicity. Tumor volume was calculated using external calipers $\left.[0.52 \times \text { (largest diameter) } \mathrm{x} \text { (smallest diameter })^{2}\right]$. Heart and body weight were also recorded at sacrifice. Results showed that voluntary wheel running reduced tumor volume per tumor [C3(1)-Sed, $422.3 \pm 89.9 \mathrm{~mm}^{3}$; C3(1)-PA, 260.2 $\pm 61.7 \mathrm{~mm}^{3}$ ] $(\mathrm{P}<0.05)$, but was associated with increased tumor number $(\mathrm{P}<0.05)$. Body composition analysis showed no differences in body fat between the groups. Heart weight/body weight ratio was increased following physical activity $(\mathrm{P}<0.05)$ providing evidence of a training effect. In conclusion, voluntary wheel running activity was effective at slowing tumor growth in the C3(1)/SV40Tag mouse model of breast cancer, but did not inhibit tumor initiation. These data provide support for further development of the C3(1)/SV40Tag mouse model for use in understanding the role of physical activity on breast cancer progression and the mechanisms for its effects.
\end{abstract}

\section{Introduction}

Breast cancer is currently the second most commonly diagnosed cancer and the leading cause of cancer related death in

Correspondence to: Dr E. Angela Murphy, Department of Pathology, Microbiology and Immunology, School of Medicine at the University of South Carolina, 6439 Garners Ferry Rd., Columbia, SC 29209, USA

E-mail: angela.murphy@uscmed.sc.edu

Key words: mammary tumors, C3(1)/SV40Tag transgenic mice, wheel running, exercise women in the United States. Epidemiological evidence indicates that physical inactivity is linked to higher breast cancer risk, whereas increased levels of physical activity in youth and throughout adulthood are associated with a reduced risk for developing breast cancer (1-4). Numerous animal studies have investigated the relationship between physical activity and reductions in mammary tumor growth and development; however, conflicting findings have weakened the basis for inferring a causal relationship. For example, many have reported benefits of exercise on mammary growth and development (5-10) while others have reported either no effect or negative outcomes (11-14). The disparate results are likely due to the variability in animal models as well as differences across exercise protocols in which intensity, mode and duration often vary making it difficult to compare across studies.

Previously, we reported that forced daily treadmill running ( $1 \mathrm{~h} / \mathrm{day}, 6$ days/week for 20 weeks) in C3(1)/SV40Tag mice significantly decreased tumor number and volume (15). To our knowledge, this was the first investigation of the effects of any type of physical activity on tumorigenesis in the C3(1)/SV40Tag mouse model of breast cancer and only the second in a transgenic breast cancer mouse model (13). The C3(1)/SV40Tag mouse is a representative model of the human disease; lesions that develop between 8-12 weeks of age are histologically similar to mammary intraepithelial neoplasia (MIN) and ductal carcinoma in situ (DCIS) observed in humans $(16,17)$. Mammary tumors develop with a $100 \%$ incidence in transgenic female mice and progress to invasive carcinomas at $\sim 16$ weeks of age making this a timely and appropriate model for interventional studies $(16,17)$.

In the present investigation, we sought to determine whether voluntary physical activity, initiated prior to the development of mammary tumors, could attenuate tumor development and growth in the triple-negative C3(1)/SV40Tag mouse model of breast cancer. These mice lack expression for estrogen receptor (ER), progesterone receptor (PR) and ERBB2 (Her2, $\mathrm{Neu}$ ), and the absence of these has been associated with poor prognosis (18). Voluntary wheel running was used as it evokes a significant training effect while minimizing stress placed on the animal since the animal self-selects its own running distance, time and speed (19). Body composition was also assessed given that reductions in body mass via increased energy expenditure or decreased energy intake (i.e. caloric restriction) have been linked to decreased breast cancer incidence (20). 
It was hypothesized that voluntary wheel running would attenuate mammary tumor incidence and growth in C3(1)/SV40Tag mice. To our knowledge, this is the only report of a reduction in tumor volume following voluntary wheel-running activity in a transgenic mouse model of breast cancer. As such, it may carry important implications for the development of specific exercise protocols for slowing the progression of incident disease. These findings may also have special relevance for women with triple negative breast tumors who are more likely to have poorer prognoses (21).

\section{Materials and methods}

Animals. Female FVB/N wild-type mice were originally purchased from Harlan Sprague-Dawley Laboratories and bred with male heterozygous C3(1)/SV40Tag transgenic mice (a gift from Dr Jeffrey Green, Chief, Transgenic Oncogenesis and Genomics Section, Laboratory of Cancer Biology and Genetics, National Cancer Institute) in the animal research facility at the University of South Carolina. Female offspring were genotyped using RT-PCR for the C3(1)/SV40Tag gene by tail snips taken prior to weaning. Mice were maintained on a 12/12-h lightdark cycle in a low-stress environment $\left(22^{\circ} \mathrm{C}, 50 \%\right.$ humidity and low noise) and provided standard rodent chow and water ad libitum. All animal care and experimentation was approved by the University of South Carolina's Institutional Animal Care and Use Committee.

Voluntary physical activity treatment. At 4 weeks of age, female C3(1)/SV40Tag and littermate (non-cancerous) FVB/N mice were randomly assigned to either the physical activity (PA) or sedentary (Sed) treatment $(n=7-15)$ [FVB-Sed, $n=7$; FVB-PA, $\mathrm{n}=13$; C3(1)-Sed, n=15; C3(1)-PA, n=12]. Additional mice were included in the FVB-PA (compared to FVB-Sed group) to increase the power for comparisons of wheel running behavior to the C3(1)-PA group. Final sample size between the C3(1) treatment groups varied as a result of misgenotyped mice that were subsequently excluded from the analysis. All mice included in the analysis survived the entire 20-week treatment period.

All mice randomized to the PA condition were housed individually and had continuous access to a voluntary running wheel (Mini-Mitter, Bend, OR, USA) from 4-24 weeks of age. Sedentary mice were individually housed in cages lacking a voluntary running wheel. Voluntary wheel running activity was measured automatically during the treatment period via a computer using Vital View physiological and behavioral monitoring software (Mini-Mitter). Total running distance, total time spent running on the wheel and peak running speed were accessed and calculated as previously described (22). Data are presented as the sum of all 2-min intervals collected each day $(24 \mathrm{~h})$ that were then averaged per week for the 20 week treatment period.

Body weight, body composition and food intake. Body weight as well as food and water intake were measured weekly throughout the treatment period. To assess potential differences in body composition that may result from the exercise intervention, a subset of animals from each group $(n=4-7)$ underwent body composition analysis at 12 weeks of age. Analysis was performed on the Lunar PIXImus X-ray densitometer (DEXA) for small animals. Animals were lightly anesthetized via isoflurane inhalation using a nose cone. The 12-week measurement point was chosen as animals had not yet developed palpable tumors; it is possible that the presence of tumors would have skewed the compositional data.

Tumor progression. Beginning at 12 weeks of age, all C3(1)/SV40Tag mice were examined twice a week for palpable tumors by the same trained investigator. The number of tumors within each mouse was recorded and the tumor volume was calculated using the formula: $0.52 \mathrm{x}$ (largest diameter) $\mathrm{x}$ (smallest diameter) $^{2}$ (23). In order to account for differences in tumor number, the total tumor volume was divided by the number of tumors within that animal. This value was then used to represent the average volume per tumor within each treatment group.

Sacrifice and tissue collection. At 24 weeks of age all mice were sacrificed via isoflurane inhalation. Visible tumors were removed from all 10 mammary glands and measured to determine tumor volume. The heart was removed and weighed to establish the effectiveness of the exercise. Spleen weight was also recorded as it has been positively associated with tumorigenesis (15).

Statistical analysis. All data were analyzed using commercial statistical software (SigmaStat, SPSS, Chicago, IL, USA). Sacrifice data including body weight, spleen weight, heart weight and DEXA data were analyzed using a two-way ANOVA (genotype $\mathrm{x}$ physical activity) with Student-Newman-Keuls post hoc analysis when appropriate. Sacrifice tumor number, volume and volume per tumor, as well as tumor latency and growth rate were analyzed using Student's t-tests. Weekly wheel running data (distance, time and speed), tumor data, body weight, food consumption and water intake were analyzed using a two-way repeated measures ANOVA (time $\mathrm{x}$ dependent variable) with Student-Newman-Keuls post hoc testing when appropriate. Statistical significance was set at an $\alpha$ value of $\mathrm{P}<0.05$. Data are presented as mean $( \pm$ SEM).

\section{Results}

Body weight, composition and food intake. Body weight was measured weekly throughout the treatment period (4-24 weeks of age) (Fig. 1). At 21 weeks of age, C3(1)-PA had significantly elevated body weight compared with FVB-PA $(\mathrm{P}<0.05)$ and from 22-24 weeks of age, both C3(1) groups (PA and Sed) had significantly greater body weight than the FVB groups (PA and Sed) $(\mathrm{P}<0.05)$. These differences however, were likely the result of the increasing tumor burden in the C3(1) mice; body weight taken at sacrifice following removal of all tumors showed no differences between the groups. Body composition assessed by DEXA also supported this finding; at 12 weeks of age there were no differences in body fat percentage and body fat mass between the groups. Percent body fat was $9.1 \pm 0.88 \%$ in the FVB-Sed mice; $10.5 \pm 0.39 \%$ in the FVB-PA mice; and $9.0 \pm 0.40$ and $9.9 \pm 0.55 \%$ in the C3(1)-Sed and C3(1)-PA mice, respectively. Total fat mass was also similar between groups; $1.68 \pm 0.19$ and $1.68 \pm 0.09 \mathrm{~g}$ in the FVB-Sed mice and C3(1)-Sed mice; and $1.83 \pm 0.08$ and $1.80 \pm 0.10 \mathrm{~g}$ in the FVB-PA and 


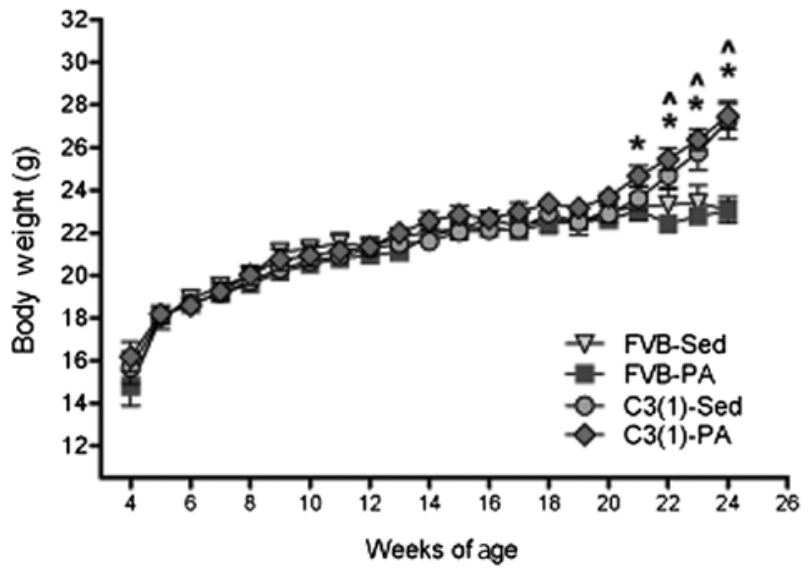

Figure 1. Body weight is not influenced by physical activity in C3(1)/SV40Tag and $\mathrm{FVB} / \mathrm{N}$ wild-type mice. Mice had access to voluntary running wheels for 20 weeks during which body weight was measured weekly. ${ }^{*}$ Significantly different from FVB-PA; ^significantly different from FVB-Sed, $\mathrm{P}<0.05$. Values are means \pm SEM

C3(1)-PA mice. As expected, food and water intake, measured weekly throughout the treatment period, was elevated in the physical activity groups $(\mathrm{P}<0.05)$ (data not shown).

Voluntary physical activity. Mice randomized to the voluntary physical activity intervention had continuous access to cage running wheels for the duration of the experiment (4-24 weeks of age). Running distance, peak speed and time increased over the first 8 weeks of the intervention (Fig. 2A, B and C, respectively) with no significant differences between the strains (FVB/N and C3(1)/SV40Tag). However, by 18-19 weeks of age distance and peak speed were significantly lower in the C3(1)/SV40Tag mice $(\mathrm{P}<0.05)$, corresponding with increasing tumor growth. Similarly, 24-h running time was significantly lower in the C3(1)/SV40Tag mice than FVB/N at 14-16 weeks and from 19-24 weeks of age $(\mathrm{P}<0.05)$. Given that wheel running distance, speed and time declined significantly in the last $\sim 4$ weeks of the experiment, we did not measure any common markers of training adaptations in the skeletal muscle as it may not accurately reflect the magnitude of change that occurred at earlier time-points during the treatment period. Therefore, we can not make any conclusions about a potential relationship between aerobic capacity and tumorigenesis in this study. It has however, been previously reported that there is not a direct effect of muscle citrate synthase activity on carcinogenesis in a rat model of MNU-induced breast carcinogenesis (24).

Effects of voluntary physical activity on tumorigenesis. Beginning at 12 weeks of age all C3(1)/SV40Tag mice were palpated twice a week for tumors, and tumor number and volume were recorded. The C3(1)-PA mice had an increase in tumor number compared to $\mathrm{C} 3(1)$-Sed mice at 22.5 weeks $(\mathrm{P}<0.05)$ and 23 weeks $(\mathrm{P}=0.07)$ (Fig. 3). Specifically at 22.5 weeks, C3(1)-Sed mice had 7.0 \pm 0.8 tumors while the C3(1)-PA mice had $8.8 \pm 1.1$ tumors. At 23 weeks these values were almost identical, but at sacrifice (24 weeks) C3(1)-Sed mice had 8.9 \pm 0.8 tumors while C3(1)-PA averaged 12.0 \pm 1.2 tumors. In addition, there was no benefit of physical activity on the time to palpa-
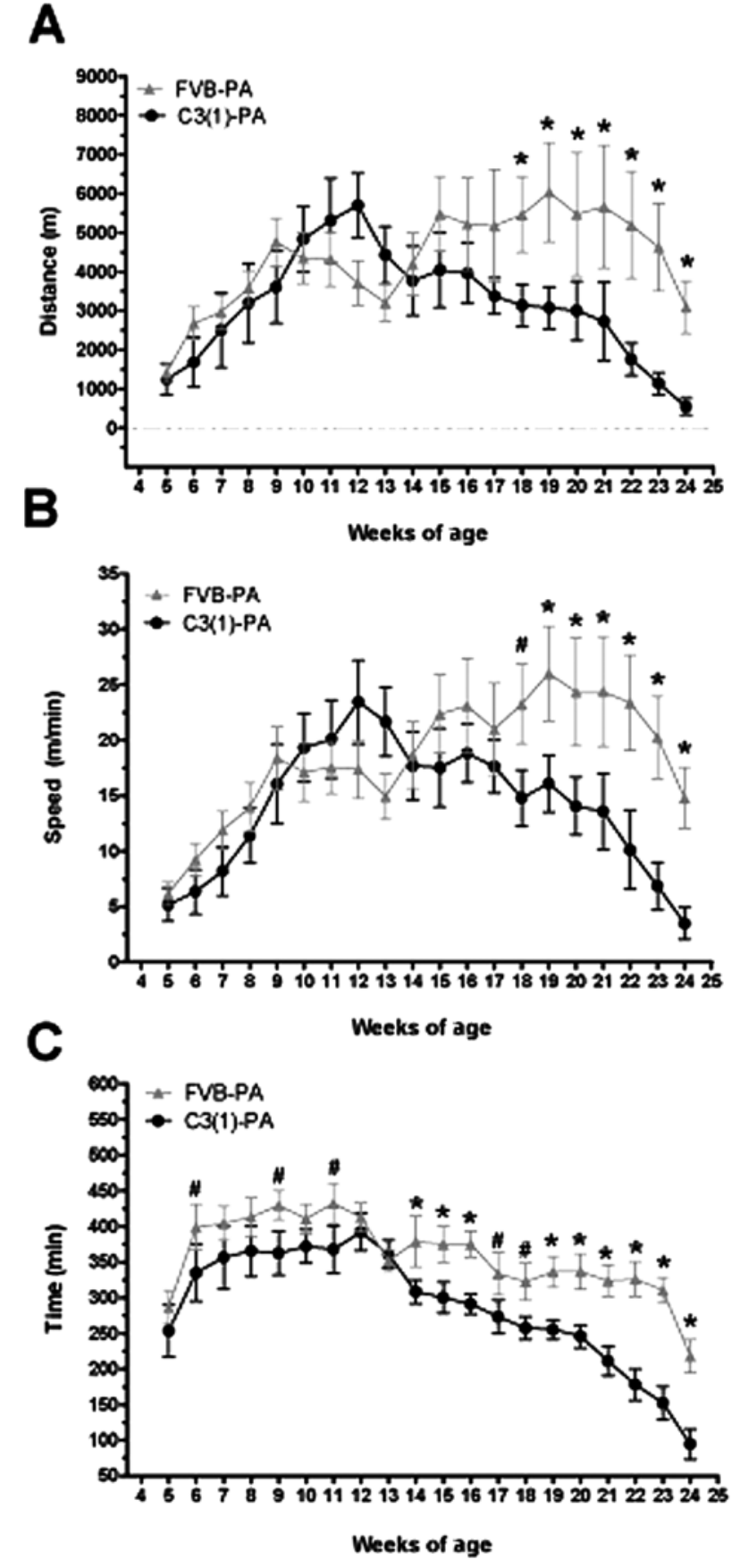

Figure 2. Mammary tumor development attenuates voluntary physical activity in C3(1)/SV40Tag mice. Mice were individually housed in cages with a voluntary running wheel. Daily (24 h) (A) running distance, (B) speed and (C) time, were averaged per week (n=12-13/group). "Significantly different from C3(1)/SV40Tag mice, $\mathrm{P}<0.05$; " trend for differences from C3(1)/SV40Tag mice. Values are means \pm SEM.

tion of the first tumor [C3(1)-Sed, $110.4 \pm 3.7$ days; C3(1)-PA, $114.0 \pm 4.9$ days].

On the contrary, average tumor volume was reduced at sacrifice (24 weeks) in the C3(1)-PA mice $\left(3,581.6 \pm 705.6 \mathrm{~mm}^{3}\right.$ compared with $4,190.8 \pm 797.9 \mathrm{~mm}^{3}$ in the sedentary mice) (Fig. 4). Furthermore, when adjusted for tumor number, average tumor volume was lower in the C3(1)-PA group at 23.5 weeks $(\mathrm{P}<0.05)$ and at sacrifice (Fig. 5). Specifically, at 23.5 weeks C3(1)-Sed had an average tumor volume per tumor of 
A

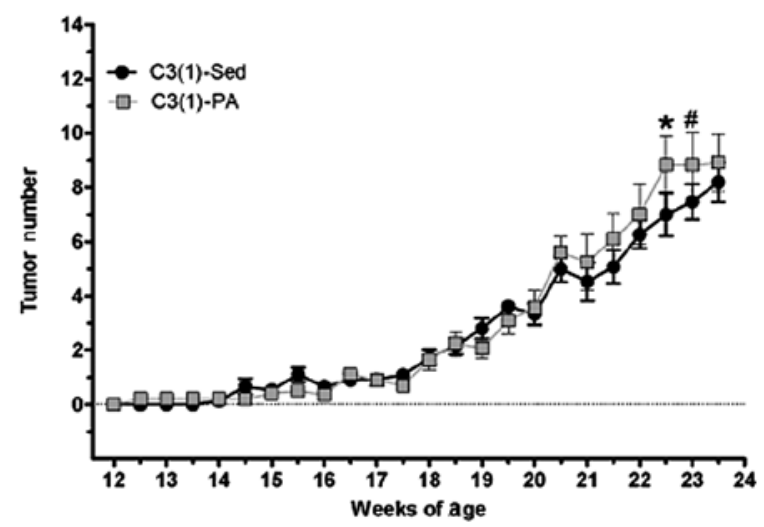

B

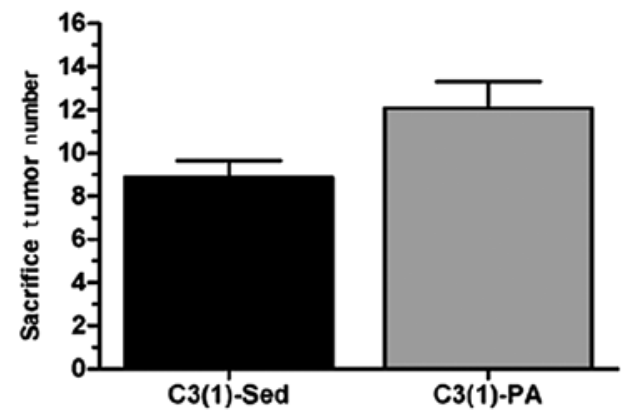

Figure 3. Physical activity increases tumor number in C3(1)/SV40Tag mice with access to a voluntary running wheel from 4 to 24 weeks of age (n=12-13/group). Tumors were counted (A) twice weekly and (B) at sacrifice. "Significantly different from C3(1)-Sed, P<0.05; " trend for differences from C3(1)-Sed, P<0.07. Values are means \pm SEM.

A

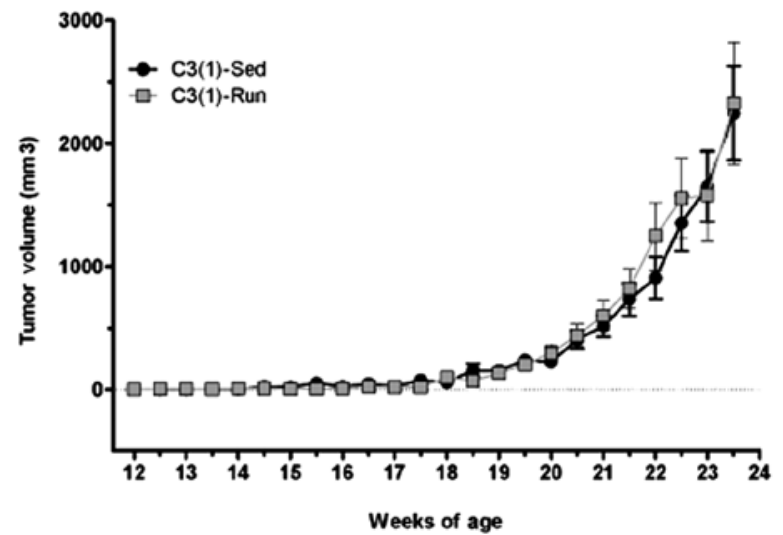

B

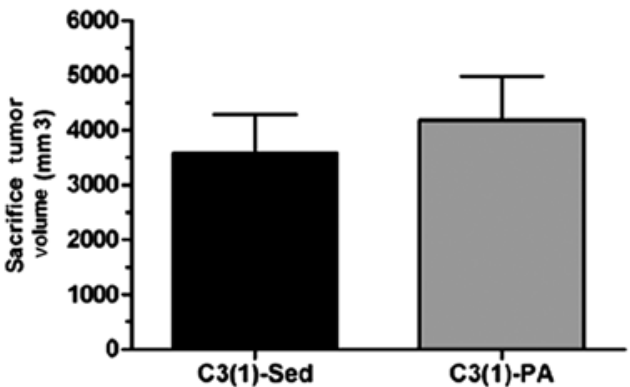

Figure 4. Physical activity does not influence tumor volume in C3(1)/SV40Tag mice with access to a voluntary running wheel from 4 weeks to 24 weeks of age ( $\mathrm{n}=12-13$ /group). Tumors were measured twice weekly (A) and at sacrifice (B) for calculation of tumor volume. Values are means \pm SEM.

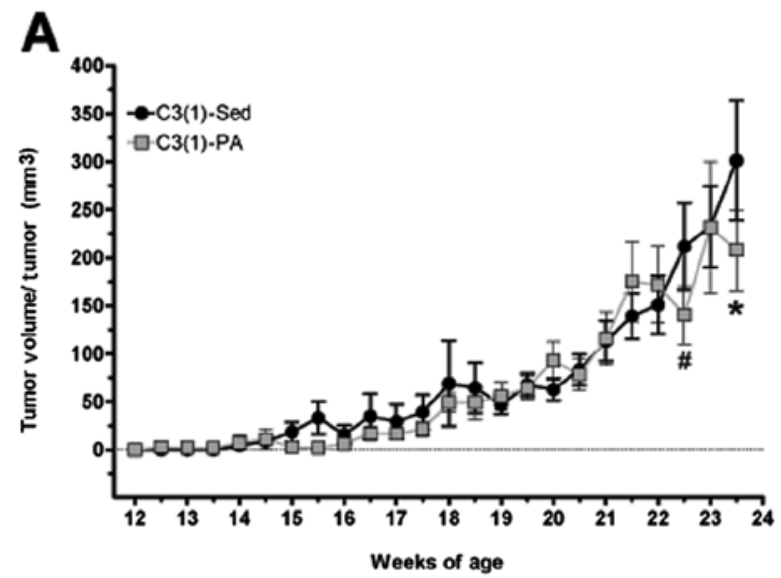

B

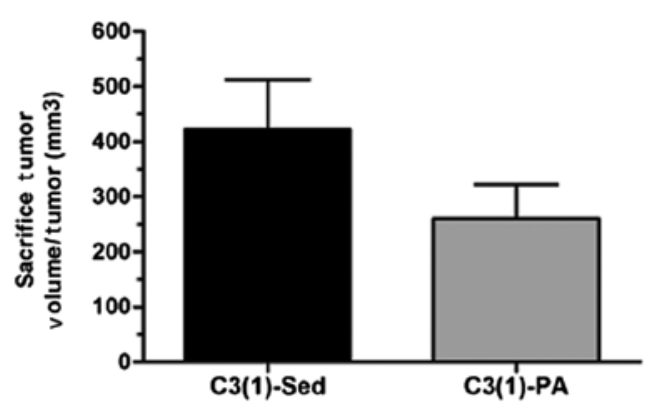

Figure 5. Physical activity decreases tumor volume expressed per tumor in C3(1)/SV40Tag mice with access to a voluntary running wheel from 4 to 24 weeks of age ( $\mathrm{n}=12-13$ /group). Volume per tumor over time is displayed in A and values at sacrifice are shown in B. "Significantly different from C $3(1)$-Sed, $\mathrm{P}<0.05$; ${ }^{*}$ trend for differences from $\mathrm{C} 3(1)$-Sed, $\mathrm{P}<0.09$. Values are means $\pm \mathrm{SEM}$. 
$301.2 \pm 62.4 \mathrm{~mm}^{3}$ compared to $207.9 \pm 42.3 \mathrm{~mm}^{3}$ in the $\mathrm{C} 3(1)-\mathrm{PA}$ mice. By sacrifice at 24 weeks of age, the average tumor volume per tumor was almost $40 \%$ lower in the C3(1)-PA mice compared to C3(1)-Sed mice as tumor volume in the C3(1)-Sed mice had risen to $422.3 \pm 89.9 \mathrm{~mm}^{3}$ versus just $260.2 \pm 61.7 \mathrm{~mm}^{3}$ in the C3(1)-PA mice. Similarly, tumor growth rate expressed as the change in tumor volume (in $\mathrm{mm}^{3}$ ) per day from time of palpation to 23.5 weeks of age was decreased $\sim 20 \%$ by physical activity [C3(1)-Sed, 9.4 $\pm 2.2 ; \mathrm{C} 3(1)-\mathrm{PA}, 7.6 \pm 1.78]$. However, this did not reach statistical significance.

Heart and spleen weight. At sacrifice heart weight was recorded as an indicator of aerobic training. A main effect of both strain and physical activity was detected when expressed relative to body weight and post hoc comparisons revealed a significant elevation in both the FVB-PA $(0.49 \pm 0.01 \%)$ and C3(1)-PA $(0.48 \pm 0.01 \%)$ groups compared with C3(1)-Sed $(0.39 \pm 0.012 \%)$ $(\mathrm{P}<0.05)$. Spleen weight was also recorded at sacrifice as this has been associated with tumor burden (15). When expressed relative to body weight, there was a main effect of strain [C3(1), $0.96 \pm 0.10 \% ; \mathrm{FVB} / \mathrm{N}, 0.40 \pm 0.005 \%]$ but no effects of exercise and no interaction.

\section{Discussion}

Epidemiological evidence supports a reduction in breast cancer risk for physically active women (2). Animal models provide a useful tool to study this relationship; however, inconsistencies among breast cancer models and physical activity protocols have hampered the reproducibility of the association reported in the epidemiological literature. Therefore we sought to establish the relationship between voluntary wheel running and tumorigenesis in the representative, triple-negative C3(1)/SV40Tag transgenic mouse model of human breast cancer. Results show that voluntary wheel running, initiated prior to tumor development, significantly reduces tumor volume per tumor independent of changes in body weight or body composition. Consistent with this, we found a slight improvement in tumor growth rate with physical activity, but this did not reach statistical significance. On the other hand however, tumor number was significantly elevated following 20 weeks of physical activity and there was no benefit of physical activity on tumor latency in this mouse model.

Several studies have examined the effects of physical activity or exercise on mammary carcinogenesis in animal models, but the results remain variable when voluntary wheel running is employed $(13,20,24,25)$. Thompson et al (26) has consistently shown decreased carcinogenic incidence after both free and motorized wheel running in the chemicallyinduced rat model of mammary tumorigenesis $(20,24,26)$. Conversely, benefits as well as no effect of exercise have been reported in mouse implantation models using MDA-MB231 human breast cancer cells $(14,25)$. However, chemical, implantation and hormone-induced cancer models may not accurately portray the development and progression of the human disease making it difficult to specifically assess the effects of exercise on disease initiation and progression. Use of genetically engineered mouse models may more closely mimic spontaneous tumor development and therefore provide clinically relevant insight into the relationship between physical activity and breast cancer growth. However, to our knowledge, only one other study has examined the effects of voluntary physical activity on breast cancer progression in a transgenic mouse model (13).

In the current investigation, we report a $\sim 40 \%$ reduction in tumor size, but no benefits on tumor establishment (measured as tumor number), following voluntary wheel running. We interpret this to mean that in the C3(1)/SV40Tag mouse, which develop tumors with $100 \%$ incidence, voluntary wheel running activity is more effective at preventing progression of tumor growth as opposed to inhibiting tumor initiation. The lack of a benefit of physical activity on tumor number is likely due to the inability of voluntary exercise to overcome the highly tumorigenic phenotype induced by the inactivation of two primary tumor suppressors, p53 and pRb, in this model (16). Alternatively, the potential for an interaction between p53 status and exercise also exists as p53, cardiovascular fitness and cancer-free survival have been linked in investigations of p53 knockout mice undergoing exercise protocols (27). Previously, Colbert et al hypothesized that their findings of increased mammary tumor incidence in $\mathrm{p} 53^{+/}$:MMTV-Wnt-1 mice with both voluntary wheel running and treadmill running may have been due to the lack of functional p53 (13). In contrast, C3(1)/SV40Tag mice express wild-type p53 which is inactivated by Tag binding. Therefore, given the role of p53 in the promotion of aerobic metabolism (vs. glycolytic), and the mitochondrial adaptive response to exercise, it is certainly plausible that these alterations in p53 may be linked to the divergent effects of exercise on tumorigenesis (28). Lastly, an interaction between the numerous physiological effects of physical activity and either C3(1) or SV40Tag expression could also have contributed to the increase in tumor number following exercise. However, such relationships were not examined in the current investigation and therefore we can only speculate that these genetic alterations of our mouse model may have contributed to the reported effects.

In contrast to our present findings, we previously showed that treadmill running reduced both tumor volume and number in C3(1)/SV40Tag mice (15). This difference is likely due to variations in the exercise protocols. In the present investigation, running distances, times and speeds declined at later ages presumably due to the increasing tumor burden and sickness. On the other hand the treadmill running protocol forced animals to maintain a constant running volume and intensity for the duration of the study. Therefore, the decline in running behavior in the current study may have occurred at a critical time for maximizing the benefits of physical activity on tumor multiplicity and/or growth in this mouse model. However, a direct comparison between these studies is somewhat hampered by allelic variation between the sets of C3(1)/SV40Tag mice as the present investigation used heterozygous mice while the treadmill study included only homozygous C3(1)/SV40Tag mice.

These results also provide evidence of a potential intensity dependent effect of exercise on tumorigenesis in C3(1)/SV40Tag mice. Previously, Thompson et al found very low intensity exercise to enhance tumorigenesis $(11,12)$, while higher intensities $(>35 \% \max )$ were related to reductions in tumor multiplicity independent of exercise duration (5). Therefore, the lack of more pronounced benefits of physical 
activity on tumorigenesis in this mouse model may be related to the decline in exercise intensity as tumors developed and progressed in size. Further experimentation including testing of additional exercise protocols and increased group sizes is necessary to determine the strength of this model for mechanistic research on the benefits of physical activity in breast cancer.

The focus of this study was not to determine biological mechanisms, however, we did assess body weight and body composition as it is well known that caloric restriction and body mass reductions decrease breast cancer risk and incidence $(6,29,30)$. Therefore, it was important to confirm that the effects of exercise were independent of an energy imbalance. We found no differences in body weight (once tumor weight was accounted for) nor body fat, between the sedentary and physically active wild-type and C3(1)/SV40Tag mice after 8 weeks of voluntary wheel running, implying that any benefits of physical activity were via mechanisms independent of differences in fat mass. Body composition analyses were only performed at 12 weeks of age in an attempt to limit the disruption of running behavior and to eliminate any potential influence of tumor composition. As a result, we can only speculate that body composition was not different between the groups as tumor burden increased. In support of our findings, several other investigations have also shown benefits of physical activity on breast cancer independent of changes in body composition $(5,9,10)$. Therefore, additional hypotheses explaining the changes induced by voluntary wheel running may include alterations in energy sensing pathways, growth related proteins and/or myokines and cytokines $(14,15,31)$. In fact, we have previously shown that treadmill exercise can reduce circulating levels of the inflammatory, pro-tumorigenic cytokines, MCP-1 and IL-6, in accordance with decreased tumor volume in C3(1)/SV40Tag mice (15). Further, exercise induced changes in the availability of metabolic fuels, due at least in part to decreased tumor blood flow, may limit tumor growth in this mouse model (31). Future studies should focus on investigating the effects of exercise on these mechanisms specifically in the C3(1)/SV40Tag mouse.

This is the first report of a benefit of voluntary wheel running on tumor growth in a transgenic mouse model of breast cancer. Although voluntary wheel running activity was associated with an increase in tumor number, the average volume per tumor was significantly reduced implying that this physical activity paradigm may be more effective at preventing tumor growth as opposed to inhibiting tumor initiation in the C3(1)/SV40Tag breast cancer mouse model. These findings contribute to the growing body of literature on the benefits of physical activity on breast cancer and provide support for the continued development of the C3(1)/SV40Tag mouse model for investigation of the relationship between breast cancer progression and physical activity.

\section{References}

1. Mittendorf R, Longnecker MP, Newcomb PA, et al: Strenuous physical activity in young adulthood and risk of breast cancer (United States). Cancer Causes Control 6: 347-353, 1995.
2. Friedenreich CM: Physical activity and breast cancer: review of the epidemiologic evidence and biologic mechanisms. Recent Results Cancer Res 188: 125-139, 2011.

3. Verloop J, Rookus MA, van der Kooy K and van Leeuwen FE: Physical activity and breast cancer risk in women aged 20-54 years. J Natl Cancer Inst 92: 128-135, 2000.

4. Thune I and Furberg AS: Physical activity and cancer risk: dose-response and cancer, all sites and site-specific. Med Sci Sports Exerc 33: S530-S610, 2001.

5. Thompson HJ, Westerlind KC, Snedden J, Briggs S and Singh M: Exercise intensity dependent inhibition of 1-methyl1-nitrosourea induced mammary carcinogenesis in female F-344 rats. Carcinogenesis 16: 1783-1786, 1995.

6. Lane HW, Teer P, Keith RE, White MT and Strahan S: Reduced energy intake and moderate exercise reduce mammary tumor incidence in virgin female BALB/c mice treated with 7,12-dimethylbenz(a)anthracene. J Nutr 121: 1883-1888, 1991.

7. Thompson HJ, Westerlind KC, Snedden JR, Briggs S and Singh M: Inhibition of mammary carcinogenesis by treadmill exercise. J Natl Cancer Inst 87: 453-455, 1995.

8. Whittal KS and Parkhouse WS: Exercise during adolescence and its effects on mammary gland development, proliferation, and nitrosomethylurea (NMU) induced tumorigenesis in rats. Breast Cancer Res Treat 37: 21-27, 1996.

9. Westerlind KC, McCarty HL, Schultheiss PC, et al: Moderate exercise training slows mammary tumour growth in adolescent rats. Eur J Cancer Prev 12: 281-287, 2003.

10. Cohen LA, Kendall ME, Meschter C, Epstein MA, Reinhardt J and Zang E: Inhibition of rat mammary tumorigenesis by voluntary exercise. In Vivo 7: 151-158, 1993.

11. Thompson HJ, Ronan AM, Ritacco KA, Tagliaferro AR and Meeker LD: Effect of exercise on the induction of mammary carcinogenesis. Cancer Res 48: 2720-2723, 1988.

12. Thompson HJ, Ronan AM, Ritacco KA and Tagliaferro AR: Effect of type and amount of dietary fat on the enhancement of rat mammary tumorigenesis by exercise. Cancer Res 49: 1904-1908, 1989.

13. Colbert LH, Westerlind KC, Perkins SN, et al: Exercise effects on tumorigenesis in a p53-deficient mouse model of breast cancer. Med Sci Sports Exerc 41: 1597-1605, 2009.

14. Jones LW, Viglianti BL, Tashjian JA, et al: Effect of aerobic exercise on tumor physiology in an animal model of human breast cancer. J Appl Physiol 108: 343-348, 2009.

15. Murphy EA, Davis JM, Barrilleaux TL, et al: Benefits of exercise training on breast cancer progression and inflammation in C3(1)SV40Tag mice. Cytokine 55: 274-279, 2011.

16. Green JE, Shibata MA, Yoshidome K, et al: The C3(1)/SV40 T-antigen transgenic mouse model of mammary cancer: ductal epithelial cell targeting with multistage progression to carcinoma. Oncogene 19: 1020-1027, 2000.

17. Maroulakou IG, Anver M, Garrett L and Green JE: Prostate and mammary adenocarcinoma in transgenic mice carrying a rat C3(1) simian virus 40 large tumor antigen fusion gene. Proc Natl Acad Sci USA 91: 11236-11240, 1994.

18. Bennett CN and Green JE: Genomic analyses as a guide to target identification and preclinical testing of mouse models of breast cancer. Toxicol Pathol 38: 88-95, 2010.

19. Davidson SR, Burnett M and Hoffman-Goetz L: Training effects in mice after long-term voluntary exercise. Med Sci Sports Exerc 38: 250-255, 2006.

20. Zhu Z, Jiang W, Zacher JH, Neil ES, McGinley JN and Thompson HJ: Effects of energy restriction and wheel running on mammary carcinogenesis and host systemic factors in a rat model. Cancer Prev Res (Phila) 5: 414-422, 2012.

21. Reis-Filho JS and Tutt AN: Triple negative tumours: a critical review. Histopathology 52: 108-118, 2008.

22. Ottenweller JE, Natelson BH, Gause WC, et al: Mouse running activity is lowered by Brucella abortus treatment: a potential model to study chronic fatigue. Physiol Behav 63: 795-801, 1998.

23. Calvo A, Yokoyama Y, Smith LE, et al: Inhibition of the mammary carcinoma angiogenic switch in C3(1)/SV40 transgenic mice by a mutated form of human endostatin. Int J Cancer 101: 224-234, 2002.

24. Mann PB, Jiang W, Zhu Z, Wolfe P, McTiernan A and Thompson HJ: Wheel running, skeletal muscle aerobic capacity and 1-methyl-1-nitrosourea induced mammary carcinogenesis in the rat. Carcinogenesis 31: 1279-1283, 2010. 
25. Welsch MA, Cohen LA and Welsch CW: Inhibition of growth of human breast carcinoma xenografts by energy expenditure via voluntary exercise in athymic mice fed a high-fat diet. Nutr Cancer 23: 309-318, 1995.

26. Thompson HJ, Wolfe P, McTiernan A, Jiang W and Zhu Z: Wheel running-induced changes in plasma biomarkers and carcinogenic response in the 1-methyl-1-nitrosourea-induced rat model for breast cancer. Cancer Prev Res (Phila) 3: 1484-1492, 2010.

27. Wang PY, Zhuang J and Hwang PM: p53: exercise capacity and metabolism. Curr Opin Oncol 24: 76-82, 2012.
28. Saleem A, Carter HN, Iqbal S and Hood DA: Role of p53 within the regulatory network controlling muscle mitochondrial biogenesis. Exerc Sport Sci Rev 39: 199-205, 2011.

29. Ligibel J: Obesity and breast cancer. Oncology (Williston Park) 25: 994-1000, 2011.

30. Cohen LA, Choi K, Backlund JY, Harris R and Wang CX: Modulation of N-nitrosomethylurea induced mammary tumorigenesis by dietary fat and voluntary exercise. In Vivo 5: 333-344, 1991

31. Thompson HJ, Jiang $\mathrm{W}$ and Zhu Z: Candidate mechanisms accounting for effects of physical activity on breast carcinogenesis. IUBMB Life 61: 895-901, 2009. 